\title{
GW46 High Voltage Isolation Switch Solid-Heat Coupling Simulation Analysis and Experimental Study
}

\author{
Yuyang Song, Benxue Liu*, Qinghui Wang, Peng Yuan \\ Zhengzhou University, Zhengzhou, Henan, 450001, China
}

\begin{abstract}
Taking the high-voltage disconnector of the GW46 type as research object, this research first use Solidworks to build its three-dimensional simplified model, then use ABAQUS to simulate the mechanical characteristics of the disconnector over a wide temperature range, analyzing the influence of the clamping force between the dynamic and static contacts on temperature, the contact pressure between the dynamic and static contacts of the high-voltage disconnector of the GW46 type increases with the ambient temperature, and the contact pressure becomes smaller. Compared simulation results with experimental data, the error is less than $5 \%$. Analysis shows that using the ABAQUS to simulation analysis of temperature rise of the high-voltage disconnector of the GW46 type, it can be determined that the contact pressure between the dynamic and static contacts changes with temperature. It avoids the complexity of the traditional theoretical calculation of query parameters and tedious calculations, and provides strong reference evidence for improving the reliability of the high-voltage disconnector of the GW46 type.
\end{abstract}

\section{Introduction}

High-voltage disconnector is the most widely used and most used high-voltage switchgear in the power grid, its usage is 2-4 times that of high-voltage circuit breakers ${ }^{[1,2]}$. Its structure includes multi-link mechanism, lock mechanism, transmission mechanism. The materials used in the structure are ordinary carbon steel, aluminum alloy, copper and other materials with different environmental temperature, when running in an open natural environment, due to the impact of various harsh environments on the product, there will be faults such as hot contact of the disconnector and stuck operation ${ }^{[3-5]}$. Mongolia in our country, the lowest temperature in local areas in winter can reach $-57.6{ }^{\circ} \mathrm{C}$, the highest temperature in Xinjiang can reach $+47^{\circ} \mathrm{C}$, the highest surface temperature is $75.8{ }^{\circ} \mathrm{C}$, the temperature of the metal body is higher, therefore, the thermal expansion and contraction of the product are very serious, product performance under extreme weather differs significantly from normal temperature performance ${ }^{[6]}$. High and low temperatures will affect the physical properties of the mechanical materials of the parts of the high-voltage disconnector, directly affects mechanical properties, indirectly affect electrical characteristics ${ }^{[7]}$. But due to the impact of the site environment, it is difficult to find the root cause of the accident, it is difficult to make timely and effective treatment. Therefore, it is necessary to carry out simulation research on the mechanical characteristics of key components, operating mechanisms, transmission mechanisms in a wide temperature range and combine products to conduct test verification.

The current research on high-voltage disconnectors mainly focuses on the cause of heating of the dynamic and static contacts ${ }^{[8,9]}$ and its online monitoring ${ }^{[10,11]}$, effect of mechanical vibration on deformation performance of high-voltage isolating switch ${ }^{[12]}$, disconnector failure ${ }^{[13,14]}$ and diagnosis ${ }^{[15]}$, research on ultra-high and low temperature of isolating switch is still blank. Therefore, using the finite element to study the mechanical characteristics of disconnectors, not only can the contact pressure between the dynamic and static contacts be changed with temperature, but also to increase its useful life, avoid unplanned outages of high-voltage equipment, improve the reliability of grid operation.

\section{Calculation model}

\subsection{Physical model}

In this article, the high-voltage disconnector of the GW46 type is taken as the research object, the outline drawing of scissors type GW46 high voltage AC isolating switch is shown in figure 1, mainly composed of main knife static contact, main gate knife, insulator, base and

\footnotetext{
*Corresponding author's e-mail:970162722@qq.com
} 
operating mechanism. Its working principle: the opening and closing movement of the disconnector is driven by the motor operating mechanism to rotate the rotary insulator, the four-bar space in the conductive box is connected to the rotating insulator through a flange, when the motor operating mechanism is operated, four links in the conductive box drive the movement of the knife brake on one side, at the same time, the cross four links connected to this drive the knife brake on the other side, so as to open and close the disconnector.

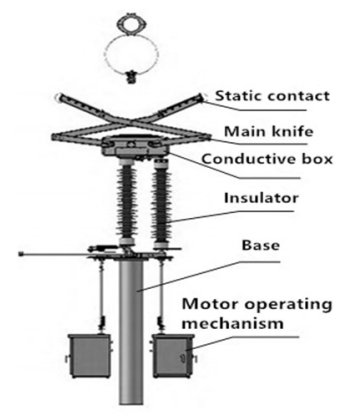

Figure 1. GW46 type high voltage isolation switch structure

Under the premise of ensuring the calculation accuracy as much as possible, Simplify the high-voltage isolation switch as follows to reduce the amount of calculation:(1) Equalizing ring system has less influence on contact pressure between dynamic and static contacts(the role of the pressure equalizing ring is to prevent side lightning, suitable for voltage form $\mathrm{AC}$, evenly distributes high pressure around objects, ensure that there is no potential difference between the various parts of the ring, so as to achieve the effect of pressure equalization), ignore equalizing ring system.(2) Keys, pins, bolts, and other parts used to connect parts can use binding constraints in the finite element model, omit the connection without considering its strength.(3) the function of the motor mechanism is to apply torque to the driving end of the high-voltage disconnect switch, torque can be applied directly in the finite element software, Ignore motorized mechanism.

\subsection{Mathematical model}

In the simulation analysis of the high-voltage disconnector of the GW46 type, problems such as redistribution of solid stress and changes in solid material properties due to temperature changes. The temperature field generated in the analysis due to these problems is non-uniform, therefore, the solid-thermal coupling method must be used to analyze the distribution of its temperature field, then determine the characteristic parameters of each point in the solid, based on this, the application of solid mechanics method to analyze its mechanical characteristics, this constitutes a complete coupling problem. The solid-thermal coupling mathematical model includes the following three aspects: temperature field equation, solid stress equation and coupling equation.

\subsubsection{Establishment of the constitutive equation under temperature field}

After the temperature of the mechanical component body of the high-voltage disconnect switch changes, due to the end of each system structure, thermal stress is generated between the internal sub-components. For high-voltage isolation switches, thermal expansion, anisotropy of structural materials, and temperature gradients in components are the main causes of thermal stress.

\subsubsection{Constitutive equation of thermal-solid field}

The thermoelastic theory of coupled field finite element analysis can be expressed as:

$$
\left\{\begin{array}{l}
\rho=\mathrm{X}^{\mathrm{e}} \rho_{0}, \\
E=\left(\mathrm{X}^{\mathrm{e}}\right)^{p} E_{0}, \\
\sigma=\lambda e+2 G \varepsilon-\beta T, \\
\tau=G \gamma,
\end{array}\right.
$$

In this formula: $\rho$ is the element density, $\rho 0$ is the initial element density; $\mathrm{Xe}$ is the relative density; $\mathrm{E}$ is the optimized relative elastic modulus; $p$ is the penalty weight factor; E0 is the initial structural stiffness of the element; $\sigma$ is the stress; $\lambda$ is the elastic modulus of the element; $\mathrm{e}$ is a finite element; $\mathrm{G}$ is the shear stress coefficient; $\varepsilon$ is the strain; $\beta$ is the thermal stress coefficient vector; $\mathrm{T}$ is the ambient temperature; $\mathrm{t}$ is the shear stress; $\gamma$ is the shear deformation.

Due to the solid-thermal coupling field, the geometrical nonlinearity and material nonlinearity of the transmission structure of the mechanism are very small,linear finite element analysis can be used in practical problem analysis. The structural governing equation of the coupled field of thermal action during operation is:

$$
\left\{\begin{array}{l}
\sigma_{i j}+b_{i j}=0, \\
\sigma_{i j}=D_{i j}\left(\varepsilon_{i j}-c_{i j} \mu_{i j}\right), \\
\varepsilon_{i j}=\frac{1}{2}\left(\mu_{i j}+\mu_{j i}\right), \\
\mu_{i j}=\bar{\mu}_{i j}, \\
\sigma_{i j}=n_{i j} \bar{t}_{i j},
\end{array}\right.
$$

In this formula: $b$ is the heat vector per unit volume generated by convective heat transfer; $\mathrm{D}$ is the structural 
elasticity matrix; $\mathrm{c}$ is the coefficient of thermal expansion tensor; $\mu$ is the structure temperature vector; $\bar{\mu}$ is a known temperature vector; $n$ is the unit vector; $\bar{t}$ is the heat flux with known boundary; stress $\sigma$ as a function of temperature field $\mu$; subscript $i$ is the number of rows of the finite element matrix, Subscript $\mathrm{j}$ is the number of columns of the finite element matrix.

Applying temperature-dependent material parameters to the model and changing temperature fields, then perform a static analysis on the high-voltage disconnect switch.

\section{Solid-thermal coupling simulation of isolation switch}

\subsection{Establishment of $3 \mathrm{~d}$ model of disconnector}

Due to the limitations of the $3 \mathrm{~d}$ modeling capabilities of ABAQUS, this article mainly uses Solidworks to build a three-dimensional solid model of the high-voltage disconnector of the GW46 type. At the beginning of modeling, for the convenience of calculation, reasonably simplify the model of GW46 isolating switch, remove structures that have less impact on analysis results, the simplified isolation switch model is shown in the figure 2 .

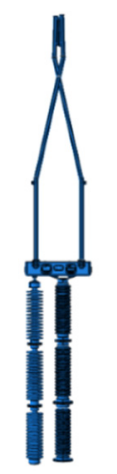

Figure 2. GW46 high voltage isolating switch finite element diagram

\subsection{Simulation of solid-heat temperature rise of disconnector}

coupling

Coupling field analysis refers to the type of analysis that involves the coupling of multiple physical fields in an analysis process.

\subsubsection{Pre-processing}

First, import the ABAQUS static analysis module by using the seamless interface between the software, set the appropriate initial conditions, solid-heat coupled temperature rise analysis. Through static analysis, the change trend of the contact pressure of the moving and static contacts of the high-voltage isolating switch with temperature can be obtained.

\subsubsection{Meshing}

Because quadratic hexahedral elements have higher calculation accuracy and efficiency, therefore, the more regular parts in this model are meshed with reduced integrals, C3D8R eight-node linear hexahedral element; use hexahedral element mesh for irregular parts as much as possible, C3D4 four-node linear tetrahedron element. The mesh number of this model is 726887 tetrahedral and hexahedral elements, grid nodes 201484.

\subsubsection{Applied loads and boundary conditions}

In practical work, the clamping force of the dynamic and static contacts of the GW46 high-voltage disconnect switch analyzed is $46 \pm 2.5 \mathrm{~kg}$ (corresponding contact pressure range is $426.3 \mathrm{~N} \sim 475.3 \mathrm{~N})$, the initial temperature is set to $14{ }^{\circ} \mathrm{C}$, make the moving contact clamping force about $449 \mathrm{~N}$, corresponds to the initial situation of the test.

\subsubsection{Analysis of simulation results}

By setting boundary conditions and loads, the change of the clamping force of the moving contact of GW46 high-voltage isolating switch with temperature is shown in table 1 .

Table 1. Statistical table of clamping force with temperature.

\begin{tabular}{cc}
\hline $\begin{array}{c}\text { Ambient temperature } \\
1{ }^{\circ} \mathrm{C}\end{array}$ & $\begin{array}{c}\text { Moving contact } \\
\text { clamping force } / \mathrm{N}\end{array}$ \\
\hline-60 & 530.5 \\
-40 & 474.1 \\
0 & 461.7 \\
+14 & 449.3 \\
+40 & 429.7 \\
+60 & 380.5 \\
\hline
\end{tabular}

Can be known from table 1: the clamping force of the moving contact decreases with increasing temperature and the clamping force of the moving contact can work normally within the range of $-40{ }^{\circ} \mathrm{C}$ to $40^{\circ} \mathrm{C}$.

\section{Comparative analysis of temperature rise test of isolating switch}

To verify the authenticity of the finite element simulation results, temperature rise test on the high-voltage disconnector of the GW46 type, the test setup is shown in figure 3. Contact contact pressure is an important parameter for high-voltage disconnectors, it is an important guarantee to maintain the conduction and non-discharge of high-voltage isolating switches ${ }^{[16]}$. Too little contact force will increase contact resistance, even the hot hair and adhesion damage, for this, a contact contact pressure detection test is required ${ }^{[17-18]}$. Environmental temperature before test $+14{ }^{\circ} \mathrm{C}$, adjust according to the rated contact pressure $46 \pm 2.5 \mathrm{~kg}$ specified by the design requirements of the high-voltage disconnector of the GW46 type, 5 temperature points (+ $60^{\circ} \mathrm{C},+20^{\circ} \mathrm{C}, 0^{\circ} \mathrm{C},-40^{\circ} \mathrm{C},-60^{\circ} \mathrm{C}$ ) ambient temperature test after meeting the requirements. 5 contact pressure cycles for each temperature point, read detection 
value, the test results and simulation results of the contact pressure of the dynamic and static contacts are shown in table 2 .

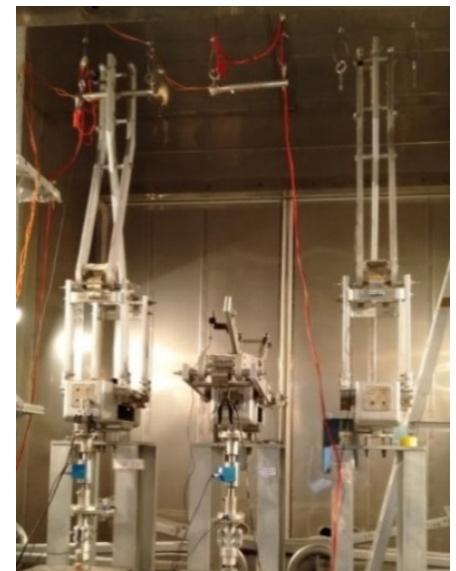

Figure 3. The high-voltage disconnector of the GW46 type test device diagram

Table 2. Comparison table of simulation and test results

\begin{tabular}{ccc}
\hline $\begin{array}{c}\text { Test ambient } \\
\text { temperature } /{ }^{\circ} \mathrm{C}\end{array}$ & $\begin{array}{c}\text { Simulation } \\
\text { results } / \mathrm{N}\end{array}$ & Test value/N \\
\hline-60 & 530.5 & 511.6 \\
\hline
\end{tabular}

\begin{tabular}{ccc}
\hline-40 & 474.1 & 469.2 \\
0 & 461.7 & 457.6 \\
+14 & 449.3 & 449.6 \\
+40 & 429.7 & 435.4 \\
+60 & 380.5 & 397.3 \\
\hline
\end{tabular}

Table 2 gives the test values of contact pressure at 6 temperatures. Can be known from table 2: The minimum average contact force at $60^{\circ} \mathrm{C}$ is $397.3 \mathrm{~N}$, less than the minimum value of its design range $426.3 \mathrm{~N}$; the maximum contact force at $-60{ }^{\circ} \mathrm{C}$ is $511.6 \mathrm{~N}$, the contact force increased by $114.3 \mathrm{~N}$ and $28.77 \%$ when the ambient temperature was $60{ }^{\circ} \mathrm{C}$, the contact pressure of the dynamic and static contacts is greatly affected by the ambient temperature, at the same time, the contact pressure between the dynamic and static contacts $511.6 \mathrm{~N}$ is greater than the maximum value of its design requirement $475.3 \mathrm{~N}$. Use the test temperature in Table 2 and the average value of the corresponding contact force test results to draw the change curve of the average contact pressure of the dynamic and static contacts of the disconnector with temperature, as shown in figure 4.

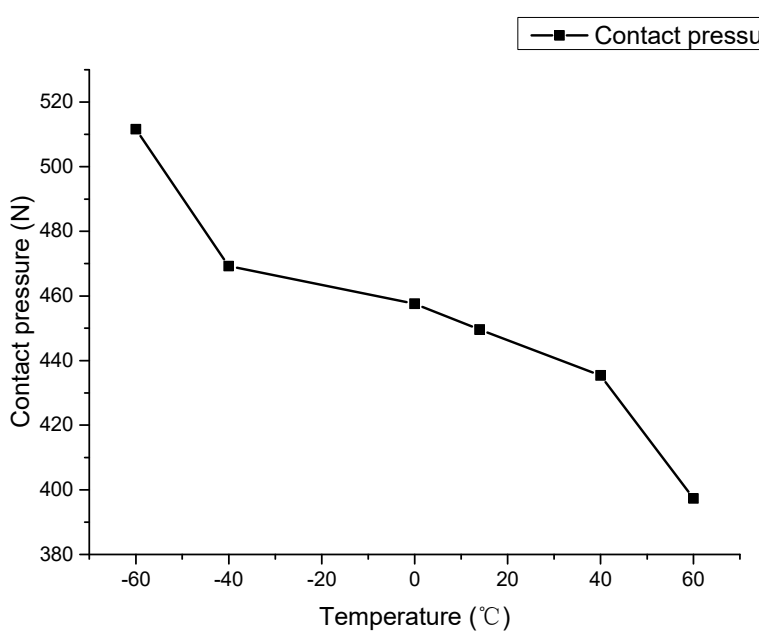

Figure 4. Test clamping force line chart

As can be seen from figure 4, the contact pressure of the dynamic and static contacts of the disconnector decreases with increasing ambient temperature. If the contact pressure measured at the temperature of $14{ }^{\circ} \mathrm{C}$ before the test is used as a reference, the contact pressure of dynamic and static contacts will decrease as the temperature rises, and the contact pressure will increase when the temperature drops. The problem of increased resistance and damaged contacts.

By comprehensively considering the relationship between the contact pressure of the dynamic and static contacts in figure 4 and the ambient temperature and the test data in table 2, it can be judged that the reasonable temperature working range of the high-voltage disconnector of the GW46 type is $-40{ }^{\circ} \mathrm{C} \sim 40{ }^{\circ} \mathrm{C}$, it is consistent with the design working temperature range $\left(-40{ }^{\circ} \mathrm{C} \sim 40{ }^{\circ} \mathrm{C}\right)$ of the high-voltage disconnector of the GW46 type.
By comparing and analyzing the simulation results and experimental data, we can know that there is a certain error between the experimental results and the simulated values, analysis of the main reasons are as follows:(1) The exact value of the material's thermal expansion coefficient cannot be determined during the solid-thermal coupling analysis using the finite element analysis method, and can only be replaced by an ideal value, which introduces a certain error.(2) Due to the simplification of the model during the pre-processing of the finite element simulation, the contact area of the dynamic and static contacts is larger than the actual situation, which causes a certain error.(3) In the actual test process, due to the influence of external environmental factors, some dust or impurities entered the dynamic and static contact system, making the dynamic and static contacts unable to effectively contact, and eventually caused some errors with the simulation results. 
The comparison between the simulation analysis results and the experimental data shows that the error between the two is within $5 \%$, which meets the accuracy requirements, and verifies the accuracy of the finite element simulation calculation.

\section{Conclusion}

(1) The solid-thermal coupling simulation analysis of the high-voltage disconnector of the GW46 type using ABAQUS is basically consistent with the results obtained through experiments, which shows that the results of ABAQUS finite element simulation analysis in actual engineering are credible.

(2) The finite element simulation analysis method can more clearly understand working situations of the high-voltage disconnector of the GW46 type, and it can identify the change trend of contact pressure between dynamic and static contacts. It provides an important way to optimize the structure and reliability design of its contact system.

\section{References}

1. Jiang Yuewen, Qian Jiaqi. (2016) Optimal selection of electrical equipment for dedicated transmission engineering based on improved ant colony algorithm [J] .Journal of Zhengzhou University (Engineering Science Edition), 37 (4): 15-19.

2. de Leon, F.,Purushothaman, S.. (2012) Heat-Transfer Model for Toroidal Transformers[J].IEEE Transactions on Power Delivery,27(2):813-820.

3. Li Ning. (2007) Research on Automatic Control Technology of High Voltage Isolation Switch in Substation [D] .Heilongjiang: Harbin Institute of Technology.

4. Liu Huanghuang, Zhang Guocan, Lin Xiangyu, et al. (2017) Analysis and Research on Thermal Failure of $110 \mathrm{kV}$ High Voltage Isolating Switch Clamps [J] .Electrical Engineering Technology, (2): 90-91.

5. Zhao Qing, Mao Dajun. (2016) Analysis of the Thermal Mechanism of Contacts of Outdoor High Voltage Disconnectors and Discussion on Measures to Prevent Overheating Faults [J] .Electrical Applications, 35 (3): 72-76.

6. Wang Xiongbo, Li Tao, Jiang Congjian, et al. (2015) Study on the electrical performance of high-voltage isolating switch for alpine trains $[\mathrm{J}]$.Locomotive Electric Drive, (1): 34-36,39.

7. Liu Lei. (2015) Preparation of Pure Silver / Silver Graphite Composite Coating for Outdoor High-Voltage Isolation Switch and Its High Temperature Performance [D] .Jiangxi: Nanchang Hangkong University.

8. Li Yong, Chen Changjie, Dong Yulin. (2016) Early Warning Method for Overheating State of High Voltage Isolation Switch Based on RBF Neural Network [J] .Automation and Instrumentation, (2): 67-69.
9. Mao Dajun, Zhao Qing, Peng Daogang. (2016) Research on Thermal Mechanism of Outdoor High Voltage Isolation Switch Contact Based on ANSYS [J] .Thermal Science and Technology, 15 (1): 46-51.

10. Huang Tao, Feng Shitao, Li Yi, et al. (2018) Research on real-time monitoring technology of contact temperature of high-voltage disconnect switch [J] .Communication Power Technology, 35 (3): 241-242.

11. Ye Zhixiang, Shen Chengbing, Sun Lixiong, et al. (2018) Extraction of Vibration Signal Features for On-line Monitoring of High Voltage Isolation Switch [J] .Yunnan Electric Power Technology, 46 (4): 95-98,102.

12. Zhou Yuqi. (2018) Research on Vibration Analysis of High-Voltage Isolation Switch Based on Vibration Analysis [D] .Jiangsu: Southeast University.

13. Liu Shibing, Ma Zhifang, Qiu Zhisheng, et al. (2019) New Strategy for Mechanical Fault Diagnosis of Catenary High Voltage Isolating Switch [J] .Journal of East China Jiaotong University, 36 (5): 136-142.

14. Chen Shigang. (2019) Research on fault diagnosis method of high-voltage isolating switch based on multi-path vibration signal fusion [D] .Beijing: Beijing Jiaotong University.

15. Zhang Yizhang, Li Shaohua, Chen Shigang, et al. (2018) Fault type and location diagnosis method of high voltage isolating switch based on ReliefF feature quantity optimization and BP neural network recognition [J] .High Voltage Apparatus, 54 (2): 12-19.

16. Huang Tao, Feng Shitao, Li Yi, et al. (2018) Research on real-time monitoring technology of contact temperature of high-voltage disconnect switch [J] .Communication Power Technology, 35 (3): 241-242.

17. Wu Xutao, Ma Bo, Li Xiuguang, et al. (2018) Simulation study on temperature field of GIS disconnectors with different voltage levels based on finite element analysis $[\mathrm{J}]$.High Voltage Apparatus, 54 (11): 160-164,169.

18. Han Bin, Lin Jiming, Chen Weijiang, et al. (2011) Effect of operating speed of disconnector on ultra-fast transient overvoltage $[\mathrm{J}]$.Chinese Journal of Electrical Engineering, 31 (31): 12-17. 